\title{
Nuclear parton density functions from dijet photoproduction at the EIC
}

\author{
M. Klasen ${ }^{*}$ and K. Kovař́̌k ${ }^{\dagger}$ \\ Institut für Theoretische Physik, Westfälische Wilhelms-Universität Münster, \\ Wilhelm-Klemm-Straße 9, D-48149 Münster, Germany
}

(Received 29 March 2018; published 11 June 2018)

\begin{abstract}
We study the potential of dijet photoproduction measurements at a future electron-ion collider (EIC) to better constrain our present knowledge of the nuclear parton distribution functions. Based on theoretical calculations at next-to-leading order and approximate next-to-next-to-leading order of perturbative QCD, we establish the kinematic reaches for three different EIC designs, the size of the parton density function modifications for four different light and heavy nuclei from He-4 over C-12 and Fe-56 to Pb-208 with respect to the free proton, and the improvement of EIC measurements with respect to current determinations from deep-inelastic scattering and Drell-Yan data alone as well as when also considering data from existing hadron colliders.
\end{abstract}

DOI: 10.1103/PhysRevD.97.114013

\section{INTRODUCTION}

Our present knowledge about the structure of hadrons at high energies is mostly encoded in parton density functions (PDFs). Since only the evolution of these quantities with the energy scale $Q$ can be calculated in perturbative $\mathrm{QCD}$, but not their dependence on the longitudinal parton momentum fraction $x$, they are generally fitted to experimental data using factorization theorems and calculations of the Wilson coefficients at next-to-leading order (NLO) and beyond [1]. The classical process for the extraction of PDFs is inclusive deep-inelastic scattering (DIS). Combined measurements of this process by the $\mathrm{H} 1$ and ZEUS experiments at DESY HERA have led to precise determinations of the proton PDFs [2].

Since the gluon density enters inclusive DIS only at NLO, other processes with leading order (LO) gluon contributions such as inclusive jet or dijet production in DIS [3] and photoproduction [4] are also important. Today, data from DESY HERA and earlier experiments are complemented by CERN LHC data on dijet, heavy-quark, and electroweak boson production [5]. Understanding the structure of the proton $(p)$ is not only an interesting research topic in its own right, but is also important to reliably estimate the production cross sections for new particles and their backgrounds [6].

\footnotetext{
michael.klasen@uni-muenster.de

†karol.kovarik@uni-muenster.de
}

Published by the American Physical Society under the terms of the Creative Commons Attribution 4.0 International license. Further distribution of this work must maintain attribution to the author(s) and the published article's title, journal citation, and DOI. Funded by SCOAP ${ }^{3}$.
For nuclei $(A)$, experimental information on their PDFs came until very recently almost exclusively from neutral and charged current fixed-target DIS as well as Drell-Yan (DY) experiments, which limited the kinematic reach to Bjorken $x$ values above about $10^{-2}$ and $Q^{2}$ values below $10^{2} \mathrm{GeV}^{2}$. The uncertainties of global nuclear PDF (nPDF) fits were therefore considerably larger than they were for protons [7]. In particular, very little is known about the gluon PDF in nuclei, which is, however, important to understand nuclear shadowing [8] and its possible relation to diffraction [9], saturation [10], and the initial condition for the creation of the quark-gluon plasma in heavy-ion collisions [11]. The situation could be somewhat improved by including pion production data from BNL RHIC [12], albeit at the cost of introducing a fragmentation function uncertainty, and recently also with the first electroweak boson [13] and in particular dijet [14] data from $\mathrm{pPb}$ collisions at the CERN LHC [15]. In addition, a recent reweighting study has shown also that forward heavy-quark and quarkonium production data from the CERN LHC have the potential to better constrain future analyses [16].

A future electron-ion collider (EIC) combining a new electron beam with the existing high-energy Relativistic Heavy-Ion Collider RHIC (eRHIC) [17] or a new ion beam with the existing high-luminosity Continuous Electron Beam Accelerator Facility at Jefferson Lab at medium energy (MEIC) [18] now offers the opportunity for measurements of nPDFs that can reach and surpass the precision known from DESY HERA. The impact of inclusive DIS has already been studied in 2012 in a White Paper [19], which was recently updated based on newer nPDFs. As shown there, an improvement of up to an order of magnitude in precision can be expected in inclusive DIS at low $x$ [20]. In a recent publication, we studied the impact of inclusive jet production measurements in DIS, i.e., of photons with large virtuality 
$Q^{2}$, at the EIC and reached similar conclusions [21] based on our previous theoretical calculations at NLO [3] and approximate next-to-next-to-leading order (aNNLO) [22]. Full NNLO calculations of inclusive jet [23] and dijet production [24] in DIS are now also available. They confirm the aNNLO results even at surprisingly large distances from the hadronic threshold and show that the NNLO corrections are moderate in size, except at the kinematical edges, and that their inclusion leads to a substantial reduction of the scale variation uncertainty on the predictions.

Here, we focus on the complementary region of almost real photons with $Q^{2} \simeq 0$. Then not only direct but also resolved photons contribute [25], so that jet photoproduction at the EIC also has the potential to finally better constrain the PDFs in the photon [26]. We consider dijet instead of inclusive jet photoproduction, so that the probed $x$ values in the heavy ion and the photon can be reconstructed (at LO exactly and beyond LO approximately) from the final state. For our numerical study, we use our established theoretical formalism of NLO calculations [4], which we have recently updated to include also aNNLO contributions [27] based on a unified approach to threshold resummation that allows us to obtain these contributions via a perturbative reexpansion [28].

We present the kinematic reach of dijet photoproduction for three different currently discussed configurations of the EIC, discuss the size of nuclear effects to be expected for different light and heavy nuclei, estimate the improvement in sensitivity on the nPDFs from the EIC with respect to current uncertainties, and establish the size of the gluon contribution in the heavy nucleus and of direct vs resolved contributions in the photon.

The remainder of the paper is organized as follows: In Sec. II we review our theoretical formalism and in Sec. III the experimental conditions that we consider. Section IV contains our main numerical results, and our conclusions and an outlook are given in Sec. V.

\section{THEORETICAL FORMALISM}

Thanks to the QCD factorization theorem [1], the differential dijet cross section in photoproduction can be expressed as

$$
\begin{aligned}
\mathrm{d} \sigma= & \sum_{a, b} \int \mathrm{d} y f_{\gamma / e}(y) \int \mathrm{d} x_{\gamma} f_{a / \gamma}\left(x_{\gamma}, \mu_{\gamma}\right) \\
& \times \int \mathrm{d} x_{A} f_{b / A}\left(x_{A}, \mu_{A}\right) \mathrm{d} \sigma_{a b}\left(\alpha_{s}, \mu_{R}, \mu_{\gamma}, \mu_{A}\right) .
\end{aligned}
$$

Here,

$$
\begin{aligned}
f_{\gamma / e}(y)= & \frac{\alpha}{2 \pi}\left[\frac{1+(1-y)^{2}}{y} \ln \frac{Q_{\max }^{2}(1-y)}{m_{e}^{2} y^{2}}\right. \\
& \left.+2 m_{e}^{2} y\left(\frac{1}{Q_{\max }^{2}}-\frac{1-y}{m_{e}^{2} y^{2}}\right)\right]
\end{aligned}
$$

is the improved Weizsäcker-Williams flux for the bremsstrahlung of photons with maximal virtuality $Q_{\max }^{2}$ and longitudinal momentum fraction $y$ from electrons with mass $m_{e}$ [29].

The photons can interact either directly, so that at LO $f_{a / \gamma}\left(x_{\gamma}, \mu_{\gamma}\right)=\delta\left(1-x_{\gamma}\right)$, or through their fluctuations into vector-meson-like quark-antiquark and gluon states described by the photon PDFs $f_{a / \gamma}\left(x_{\gamma}, \mu_{\gamma}\right)$. Beyond LO, both contributions are related through the factorization of collinear singularities. We use the Glück-Reya-Vogt NLO parametrizations of the photon PDFs [30] obtained in the perturbatively stable DIS $\gamma$ scheme [31]. These PDFs can be transformed to the $\overline{M S}$ factorization scheme via

$$
f_{q / \gamma}^{\mathrm{MS}}\left(x_{\gamma}, \mu_{\gamma}\right)=f_{q / \gamma}^{\mathrm{DIS}_{\gamma}}\left(x_{\gamma}, \mu_{\gamma}\right)-\frac{\alpha}{2 \pi} e_{q}^{2} C_{\gamma}\left(x_{\gamma}\right),
$$

i.e., through the absorption of the pointlike Wilson coefficient in the photon structure function

$C_{\gamma}(x)=3\left[\left(x^{2}+(1-x)^{2}\right) \ln \frac{1-x}{x}+8 x(1-x)-1\right]$

into the PDFs of quarks with fractional charge $e_{q}$ in the photon. Subsequently, other NLO parametrizations of the photon PDFs in the DIS ${ }_{\gamma}$ [32] and M S scheme [33] have been proposed. In the absence of experimental constraints, their spread must be considered a contribution to the theoretical uncertainty that a future EIC might also help to reduce [26]. As we will see, the constraints on nuclear and photon PDFs come from complementary kinematic regions.

For the nuclear PDFs $f_{b / A}\left(x_{A}, \mu_{A}\right)$, we consider the nCTEQ15 NLO fits with their intrinsic nuclear mass dependence and 32 associated error PDFs as our baseline, and we estimate the impact of the inclusive pion production data from BNL RHIC with their nCTEQ15-np variants [12]. In addition, we will show results using the more recent EPPS16 NLO fits, which are based on the factorized form

$$
f_{b / A}\left(x_{A}, \mu_{A}\right)=R_{b / A}\left(x_{A}, \mu_{A}\right) f_{b / p}\left(x_{A}, \mu_{A}\right),
$$

and use information on the nuclear modification factor $R_{b / P b}$ from $\mathrm{pPb}$ collisions at the CERN LHC and CT14 NLO free proton PDFs $f_{b / p}\left(x_{A}, \mu_{A}\right)$ [5].

The partonic cross sections $\mathrm{d} \sigma_{a b}\left(\alpha_{s}, \mu_{R}, \mu_{\gamma}, \mu_{A}\right)$ are well known at NLO [4]. We have recently included aNNLO corrections [27] based on a unified approach to NNLO soft and virtual corrections from a reexpansion of all-order resummation [28]. These corrections dominate close to partonic threshold

$$
z=\frac{\left(p_{1}+p_{2}\right)^{2}}{\left(p_{a}+p_{b}\right)^{2}} \rightarrow 1,
$$

i.e., when the invariant mass of the dijet pair with fourmomenta $p_{1,2}$ approaches the one of the incoming partons 
with four-momenta $p_{a, b}$. For brevity, we present here only the master formula at NLO

$$
\begin{aligned}
\mathrm{d} \sigma_{a b}= & \mathrm{d} \sigma_{a b}^{B} \frac{\alpha_{s}\left(\mu_{R}\right)}{\pi}\left[c_{3} D_{1}(z)+c_{2} D_{0}(z)+c_{1} \delta(1-z)\right] \\
& +\frac{\alpha_{s}^{d_{\alpha_{s}}+1}\left(\mu_{R}\right)}{\pi}\left[A^{c} D_{0}(z)+T_{1}^{c} \delta(1-z)\right]
\end{aligned}
$$

where the + distributions

$$
D_{l}(z)=\left[\frac{\ln ^{l}(1-z)}{1-z}\right]_{+}
$$

denote leading, next-to-leading logarithms, etc., and $d_{\alpha_{s}}=$ $0,1,2, \ldots$ the power in the strong coupling constant $\alpha_{s}$ of the underlying Born cross section $\mathrm{d} \sigma_{a b}^{B}$. For a simple color flow, the second part of the equation is absent. The master formula at NNLO and further details can be found in Ref. [28]. For pair-invariant mass kinematics and in the $\bar{M} S$ scheme, the coefficients for a simple color flow read

$$
\begin{aligned}
& c_{3}=C_{F}-N_{C}, \\
& c_{2}=C_{F}\left[-\ln \left(\frac{\mu_{A}^{2}}{s}\right)-\frac{3}{4}+2 \ln \left(\frac{-u}{s}\right)\right]+N_{C} \ln \left(\frac{t}{u}\right)-\frac{\beta_{0}}{4} \\
& c_{1}^{\mu}=-\frac{3 C_{F}}{4} \ln \left(\frac{\mu_{A}^{2}}{s}\right)+\frac{\beta_{0}}{4} \ln \left(\frac{\mu_{R}^{2}}{s}\right)
\end{aligned}
$$

with $C_{F}=4 / 3, N_{C}=3, \beta_{0}=\left(11 N_{C}-2 n_{f}\right) / 3, n_{f}$ quark flavors, and the usual Mandelstam variables $s, t$, and $u$ for the QCD Compton process $\gamma q \rightarrow q g$, and

$$
\begin{aligned}
& c_{3}=2\left(N_{C}-C_{F}\right), \\
& c_{2}=-\frac{3 C_{F}}{2}+N_{C}\left[-\ln \left(\frac{\mu_{A}^{2}}{s}\right)+\ln \left(\frac{t u}{s^{2}}\right)\right], \\
& c_{1}^{\mu}=-\frac{\beta_{0}}{4} \ln \left(\frac{\mu_{A}^{2}}{s}\right)+\frac{\beta_{0}}{4} \ln \left(\frac{\mu_{R}^{2}}{s}\right)
\end{aligned}
$$

for photon-gluon fusion $\gamma g \rightarrow q \bar{q}$ [27]. For a complex color flow,

$$
\begin{aligned}
c_{3}= & 2 C_{F}, \\
c_{2}= & -C_{F} \ln \left(\frac{\mu_{\gamma}^{2}}{s}\right)-C_{F} \ln \left(\frac{\mu_{A}^{2}}{s}\right)-\frac{11}{2} C_{F}, \\
c_{1}^{\mu}= & -C_{F}\left[\ln \left(\frac{-t}{s}\right)+\frac{3}{4}\right] \ln \left(\frac{\mu_{\gamma}^{2}}{s}\right)-C_{F}\left[\ln \left(\frac{-u}{s}\right)+\frac{3}{4}\right] \\
& \times \ln \left(\frac{\mu_{A}^{2}}{s}\right)+\frac{\beta_{0}}{2} \ln \left(\frac{\mu_{R}^{2}}{s}\right)
\end{aligned}
$$

for quark-(anti)quark scattering $q q^{\prime} \rightarrow q q^{\prime}$ and $q \bar{q}^{\prime} \rightarrow q \bar{q}^{\prime}$ and similarly for the other partonic processes [28]. Note that in the coefficients of the resolved processes also the photon factorization scale $\mu_{\gamma}$ enters and that the coefficients $c_{1}^{\mu}$ contain only the scale-dependent parts, whereas their finite parts must be taken from our full NLO calculation [4].

The size of the aNNLO corrections has been shown not to exceed $+7 \%(-7 \%)$ at large jet transverse momentum $p_{T}$ and forward (backward) rapidity $\eta$ [27]. More important is the reduction of the scale uncertainty in particular at large $p_{T}$, which strengthens our confidence in the perturbative calculation. Strictly speaking, the aNNLO formalism described above applies to massless jets [34], whereas experimentally jets are defined with an algorithm and have nonvanishing mass. Work on implementing the jet mass corrections is currently in progress. Their impact is expected to be small, in particular when the jet radius $R=1$, so that $\ln R$ terms vanish.

\section{EXPERIMENTAL CONDITIONS}

Several variants of the EIC are currently under debate. The eRHIC version proposed at BNL would add a new electron ring with energy $E_{e}=16-21 \mathrm{GeV}$ to the existing ion beam with energy $E_{A}=100 \mathrm{GeV}$, so that a total center-of-mass energy per nucleon of $\sqrt{s}=80-90 \mathrm{GeV}$ and an annual integrated luminosity of about $\mathcal{L}=10 \mathrm{fb}^{-1}$ could be reached. At Jefferson Lab, the MEIC planning is built on the existing electron ring with energy $E_{e}=12 \mathrm{GeV}$ and would add to it an ion beam of energy $E_{A}=40 \mathrm{GeV}$, resulting in a lower center-of-mass energy of $\sqrt{s}=45 \mathrm{GeV}$, but a higher integrated luminosity of $\mathcal{L}=100 \mathrm{fb}^{-1}$. We will therefore consider all three collider scenarios.

The maximum virtuality $Q_{\max }^{2}$ and longitudinal momentum fraction $y$ of the photon can be determined either from the (anti)tagged scattered electron or from the hadronic final state with the Jacquet-Blondel method, which has proven advantageous at very low values of $y$ at DESY HERA. Current detector designs aim at $Q^{2}<0.1 \mathrm{GeV}^{2}$ and $0.01 \leq y \leq 0.95$. The electromagnetic calorimeter would span the rapidity range $-4<\eta<4$ [19]. No specifications have so far been fixed for the hadronic calorimeter, so that we assume the same coverage. In the following section we will, however, see that a hadronic calorimeter of size $-1<\eta<3$ would be sufficient for the jet measurements proposed here. We assume that the jets are reconstructed with an anti- $k_{T}$ algorithm, a distance parameter $R=1$ in the $\eta-\phi$ plane, and a massless $p_{T}$ recombination scheme [35]. Similar to our study of inclusive jet production in DIS [21], we assume that jets can be measured down to transverse momenta of $p_{T} \geq 5(4.5) \mathrm{GeV}$, where the cuts on the leading (subleading) jet must be sufficiently different to avoid sensitivity to soft gluon radiation [36]. We then set all scales to the average transverse momentum $\mu_{R, \gamma, A}=\bar{p}_{T}=$ $\left(p_{T, 1}+p_{T, 2}\right) / 2$.

\section{NUMERICAL RESULTS}

We now present our numerical results for dijet photoproduction at an EIC. In particular, we compute 
single-differential cross sections in the average momentum $\bar{p}_{T}$ and rapidity $\bar{\eta}=\left(\eta_{1}+\eta_{2}\right) / 2$ of the two jets as well as in the deduced initial parton momentum fractions

$$
\begin{aligned}
x_{A}^{\mathrm{obs}} & =\frac{p_{T, 1} e^{\eta_{1}}+p_{T, 2} e^{\eta_{2}}}{2 E_{A}} \\
\text { and } \quad x_{\gamma}^{\mathrm{obs}} & =\frac{p_{T, 1} e^{-\eta_{1}}+p_{T, 2} e^{-\eta_{2}}}{2 y E_{e}}
\end{aligned}
$$

in the nucleus $A$ and the photon $\gamma$.

\section{A. Dijet photoproduction at different EICs}

Figure 1 shows the kinematic reaches of the three EIC variants discussed in the previous section. Average transverse momenta (top left) of up to 10 and $20 \mathrm{GeV}$ can be reached at MEIC and eRHIC, respectively, extending the range in the probed scales by up to a factor of 4 from 100 to $400 \mathrm{GeV}^{2}$. At the largest $\bar{p}_{T}$, between $10^{3}$ and 10 events
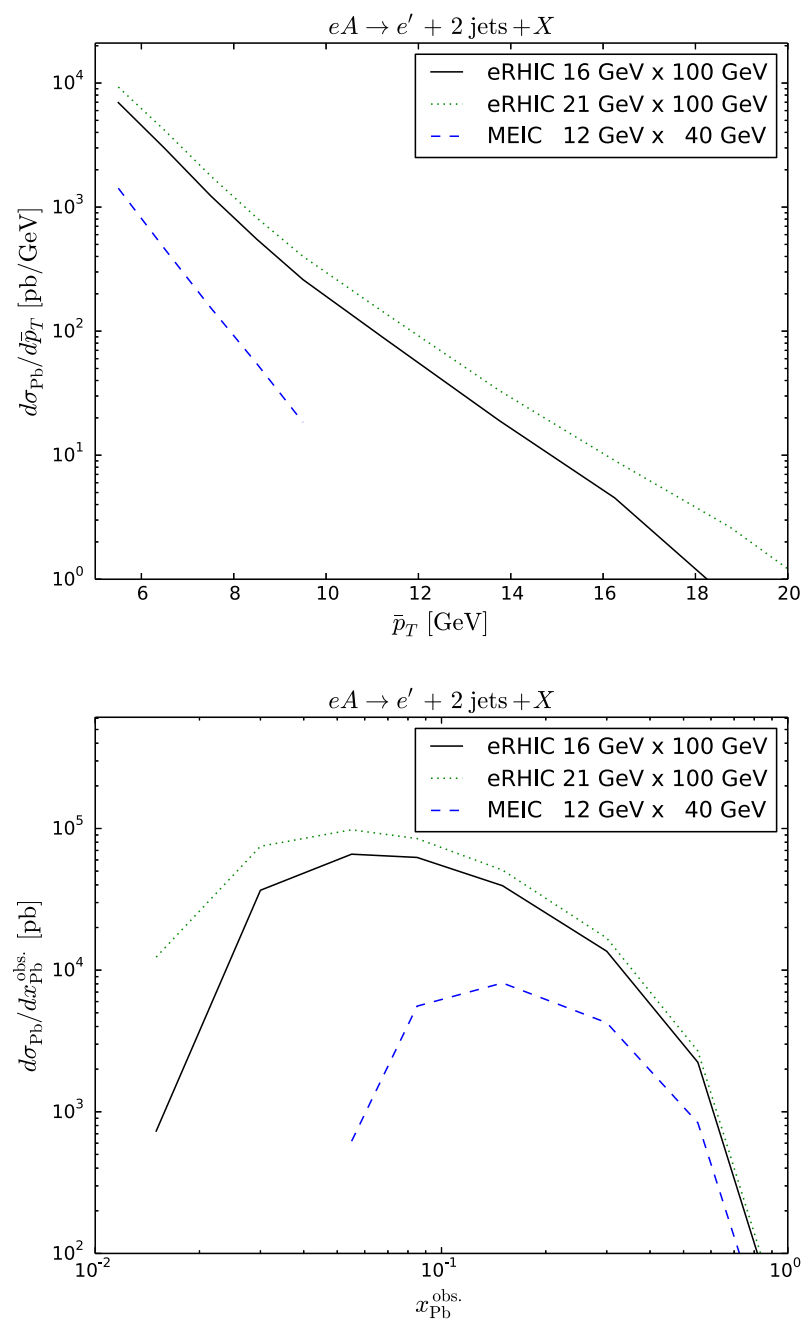

would be collected annually with luminosities of 100 or $10 \mathrm{fb}^{-1}$. Compared to inclusive jet production in DIS, where $p_{T}^{2}$ and $Q^{2}$ values of up to $10^{3} \mathrm{GeV}^{2}$ are kinematically accessible [21], the scales probed in dijet photoproduction are therefore more limited.

Similar conclusions can be drawn for the $x$ values probed in the ion (bottom left) and the photon (bottom right). At the MEIC, they cover the region of the European Muon Collaboration (EMC) effect [37] above $10^{-1}$ and antishadowing [38] above a few $10^{-2}$, but do not reach into the shadowing region below this value $[8,9]$. The fact that the photon PDFs are only probed at larger values of $x$ above a few $10^{-2}$, where they are dominated by the pointlike (quark) contribution [25] and gluon-initiated contributions are small [26], is advantageous, as it reduces the photon PDF uncertainty on the determination of the nuclear PDFs.

The two jets are produced with average rapidities (top right) between -1 and 2 or 3 at the MEIC or eRHIC. The ion beam is assumed to move in the positive $z$ direction
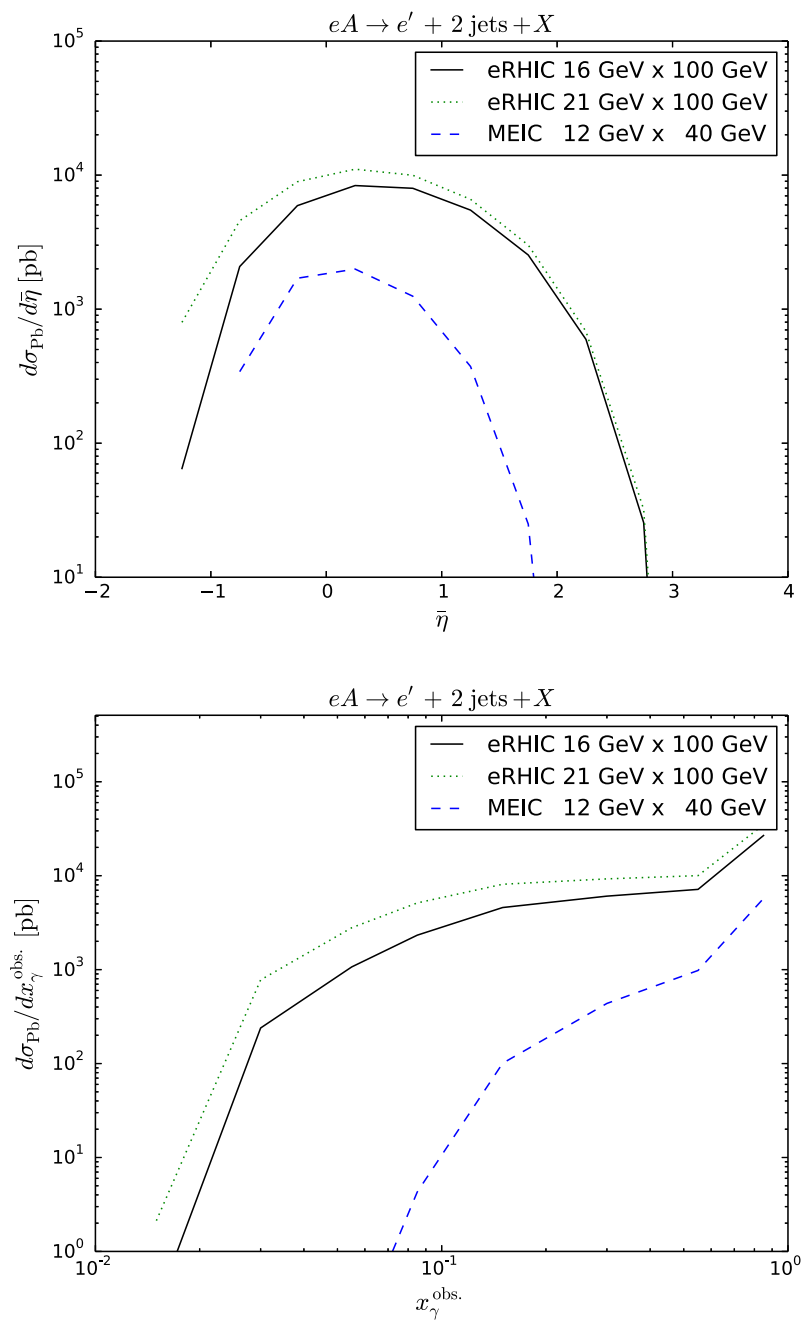

FIG. 1. Dijet photoproduction in electron-lead ion collisions at eRHIC and MEIC with electron beam energies of 12 to $21 \mathrm{GeV}$ and ion beam energies per nucleon of 40 to $100 \mathrm{GeV}$. Shown are differential cross sections in the average jet transverse momentum (top left), average rapidity (top right), and observed parton momentum fractions in the probed nucleon (bottom left) and photon (bottom right). 
similar to DESY HERA. This shows that a hadronic calorimeter with this coverage would be sufficient to measure dijet photoproduction.

\section{B. Dijet photoproduction on different nuclei}

In this and the following subsections, we present ratios $R_{A} / R_{p}$ of electron-ion over electron-proton cross sections as functions of the same kinematic variables as above in order to study the sensitivity of the EIC measurements on nuclear effects [39]. These ratios also have the advantage of further reducing unphysical scale uncertainties. We concentrate on the eRHIC design with a center-of-mass energy per nucleon of $\sqrt{s}=80 \mathrm{GeV}$. First, we study in Fig. 2 the size of these effects for different light and heavy nuclei from $\mathrm{He}-4$ (dot-dashed red lines) over C-12 (dotted green lines) and Fe-56 (dashed blue lines) to $\mathrm{Pb}-208$ (full black lines), based on the central nCTEQ15 fit. Generally speaking, the difference to bare protons increases with the nuclear mass from a few percent up to a factor of 2 . It changes sign from low to high $\bar{p}_{T}$ (top left) and twice in the other distributions. The distribution in $x_{A}^{\text {obs }}$ (bottom left) clearly shows the regions of the EMC effect, antishadowing and also shadowing at large, intermediate, and small $x$, which are correlated with the backward, central, and forward regions in $\bar{\eta}$ (top right). The distribution in $x_{\gamma}^{\text {obs }}$ (bottom right) shows that direct and pointlike photons, which are well constrained, probe not only the shadowing but also the antishadowing region. On top of the prediction for lead ions, we show simulated EIC data with a total systematic error of $2 \%$ (black error bars), which is expected to dominate over the statistcal error (cf. Fig. 3.25 of Ref. [19]).
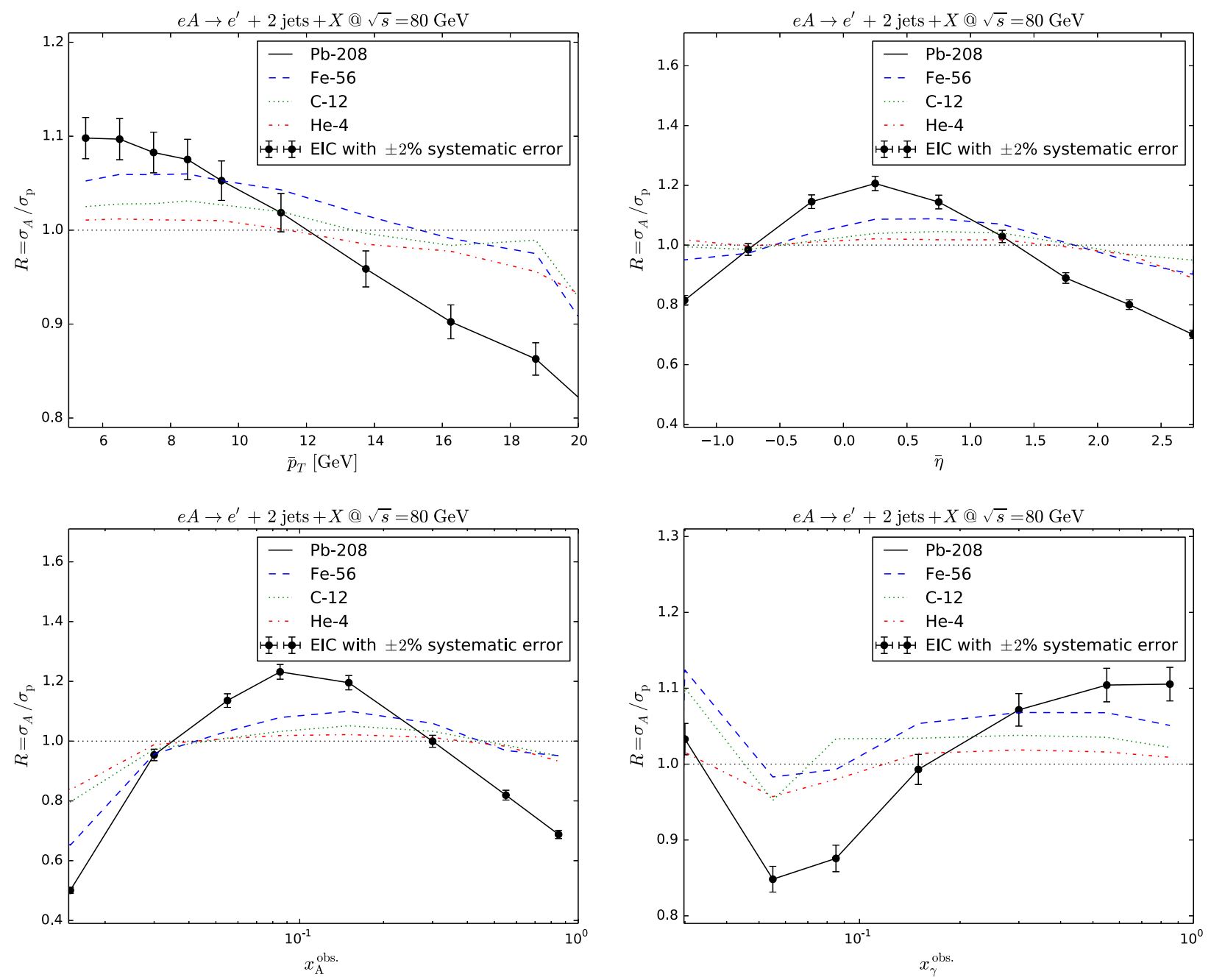

FIG. 2. Dijet photoproduction in electron-ion collisions with beam energies of 16 and $100 \mathrm{GeV}$, respectively, at eRHIC for different nuclei: $\mathrm{Pb}-208$ (full black lines), Fe-56 (dashed blue lines), C-12 (dotted green lines), and He-4 (dot-dashed red lines). Shown are the ratios of electron-ion over electron-proton cross sections as a function of the average jet transverse momentum (top left), average rapidity (top right), and observed parton momentum fractions in the probed nucleon (bottom left) and photon (bottom right). Error bars indicate the expected experimental precision. 


\section{Sensitivity to nuclear parton density functions}

In Fig. 3 we focus on the predictions for $\mathrm{Pb}-208$ and include the current uncertainty of the nCTEQ15-np fit (red shaded bands), where no constraints (and uncertainties) from pion production at BNL RHIC are included. With the information from DIS and DY data alone, all four distributions are consistent with unity within errors almost everywhere. This clearly demonstrates the need for improvements on the nuclear PDFs. The uncertainties increase with average transverse momentum $\bar{p}_{T}$ and toward small values of $x_{\gamma}^{\text {obs }}$, i.e., toward very large values of $x_{A}^{\text {obs }}$, while they are rather uniformly distributed elsewhere. It is clear that the EIC measurements (black error bars) represent an improvement of up to an order of magnitude compared to nCTEQ15-np. The direct contribution (dot-dashed orange line) increases as expected toward large $\bar{p}_{T}$, in the backward rapidity region and small $x_{A}^{\mathrm{obs}}$, and is contained in the highest $x_{\gamma}^{\mathrm{obs}}$ bin. On the other hand, the gluon in the lead ion (dashed blue line) contributes most at small $\bar{p}_{T}$ and $x_{A}^{\text {obs }}$, i.e., in the shadowing region, and again in the backward region and at large $x_{\gamma}^{\mathrm{obs}}$.

When the pion data from BNL RHIC are included, the nCTEQ15 uncertainties are of course smaller, as it can be seen from Fig. 4. They are then similar in size to those from EPPS16 (green shaded bands), although one must keep in mind that these two analyses are based on quite different theoretical assumptions. It is interesting to see that they nevertheless overlap to a rather good degree. Even after the inclusion of BNL RHIC pion data [40] in nCTEQ15 and CERN LHC, in particular CMS dijet data [41], in EPPS16, there is still substantial room for improvement from the
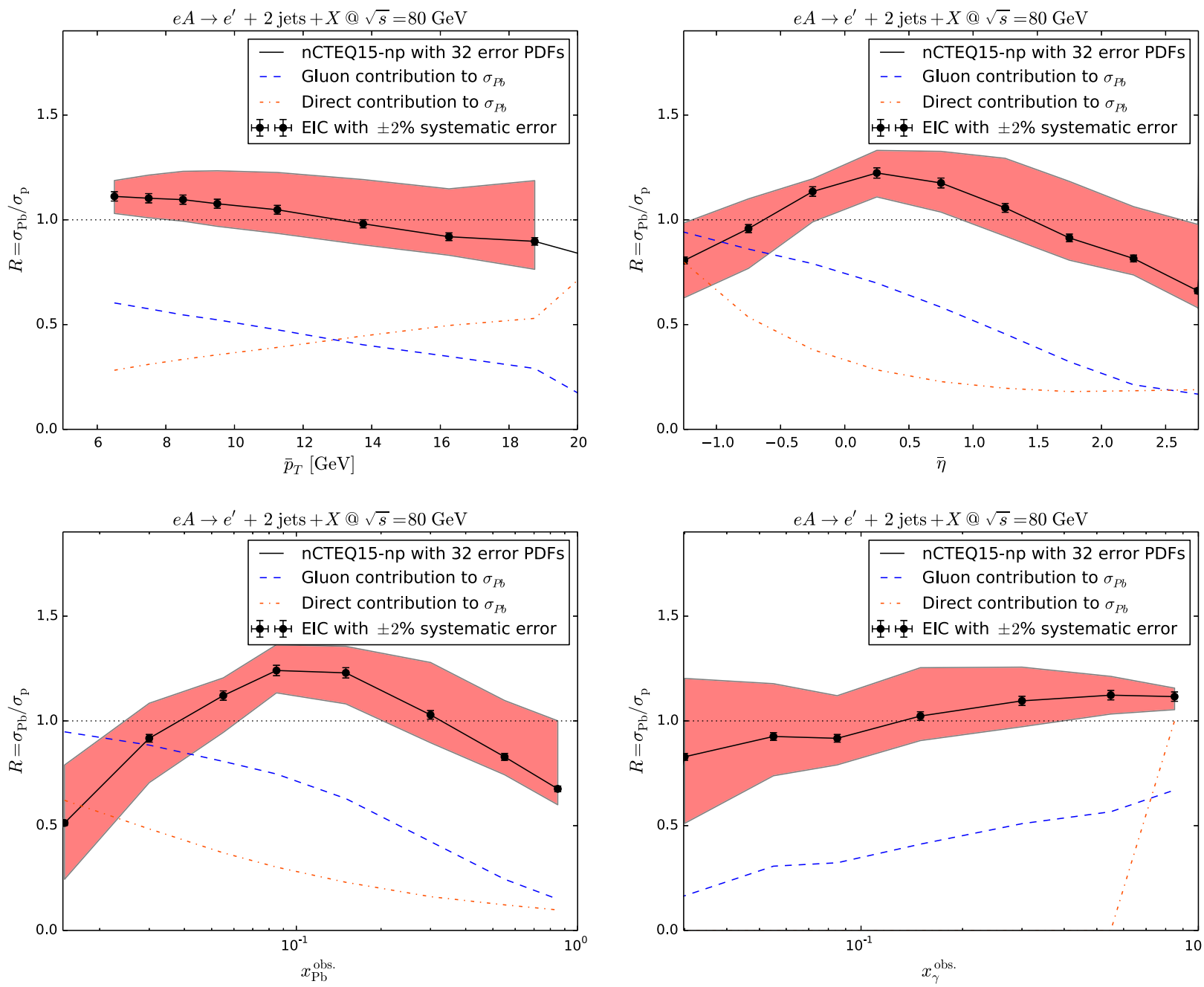

FIG. 3. Dijet photoproduction in electron-lead ion collisions with beam energies of 16 and $100 \mathrm{GeV}$, respectively, at eRHIC. Shown is the ratio of electron-lead ion over electron-proton cross sections (full black lines) including the current nuclear PDF uncertainty from the nCTEQ15 fit to DIS and DY data only (red-shaded bands) as well as the relative gluon contribution in the lead ion (dashed blue lines) and the direct photon contribution (dot-dashed orange) to the total cross section as a function of the average jet transverse momentum (top left), average rapidity (top right), and observed parton momentum fractions in the probed nucleon (bottom left) and photon (bottom right). Error bars indicate the expected experimental precision. 

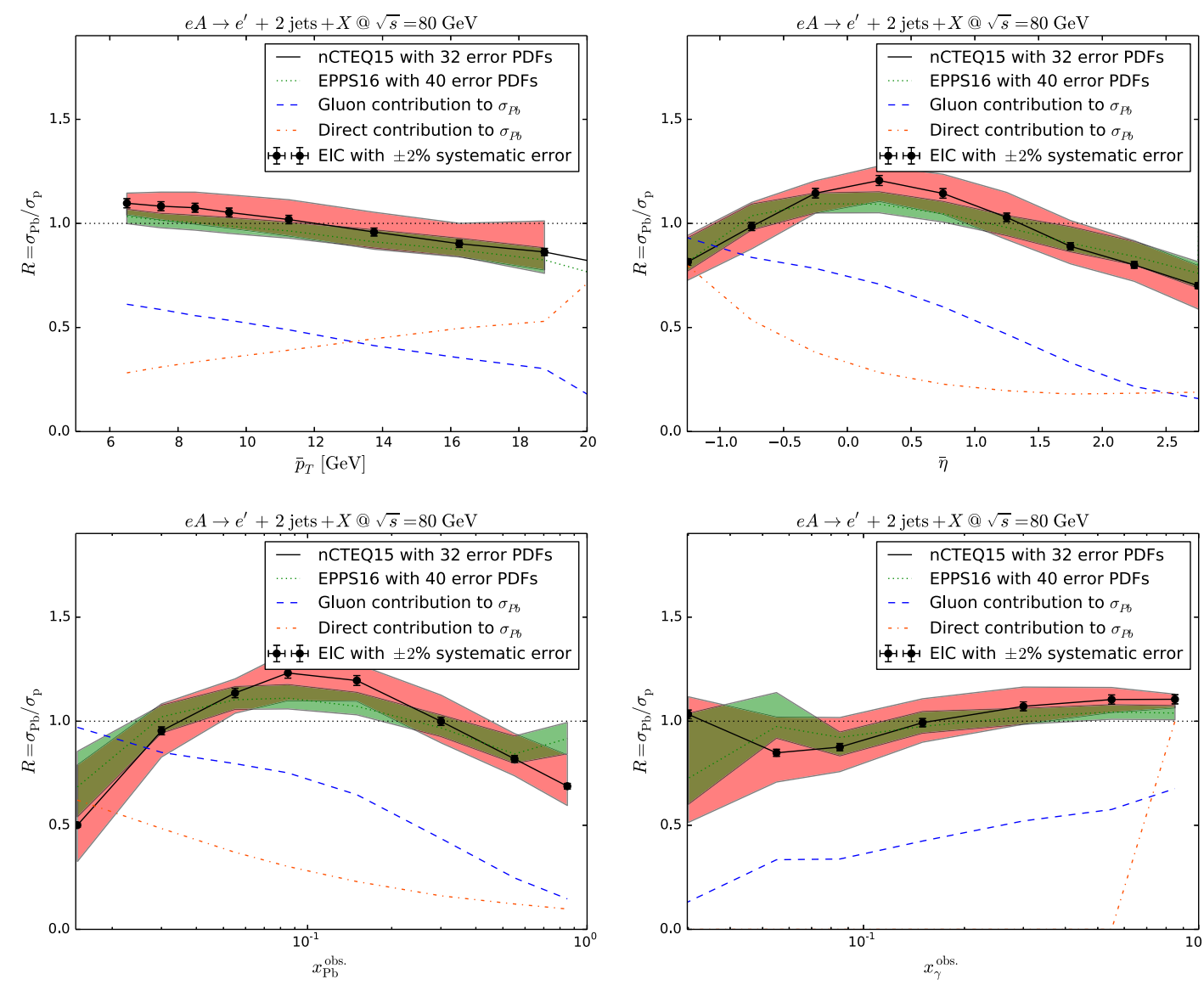

FIG. 4. Same as Fig. 3 for the nCTEQ15 fit including also inclusive pion data from D-Au collisions at BNL RHIC, and for the central EPPS16 fit (dotted green lines) to, in particular, dijet data from the CERN LHC as well as the corresponding (green-shaded) error bands.

EIC, as the simulated data have error bars that are still by about a factor of 5 smaller than the current theoretical uncertainties.

The current knowledge (or prejudice) on the nuclear size dependence of the PDFs is presented in Fig. 5 as ratios of

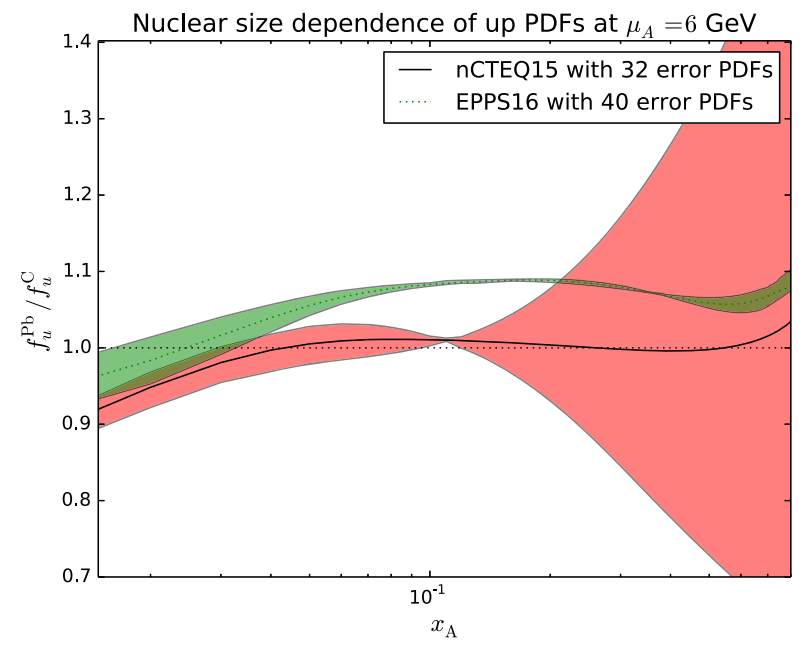

$\mathrm{Pb}-208$ over C-12 PDFs for up quarks (left) and gluons (right) as a function of parton momentum fraction $x_{A}$ and at a factorization scale $\mu_{A}=6 \mathrm{GeV}$ relevant for the cross sections discussed in this paper. The DIS data on different targets, in particular from the CERN NMC experiment,

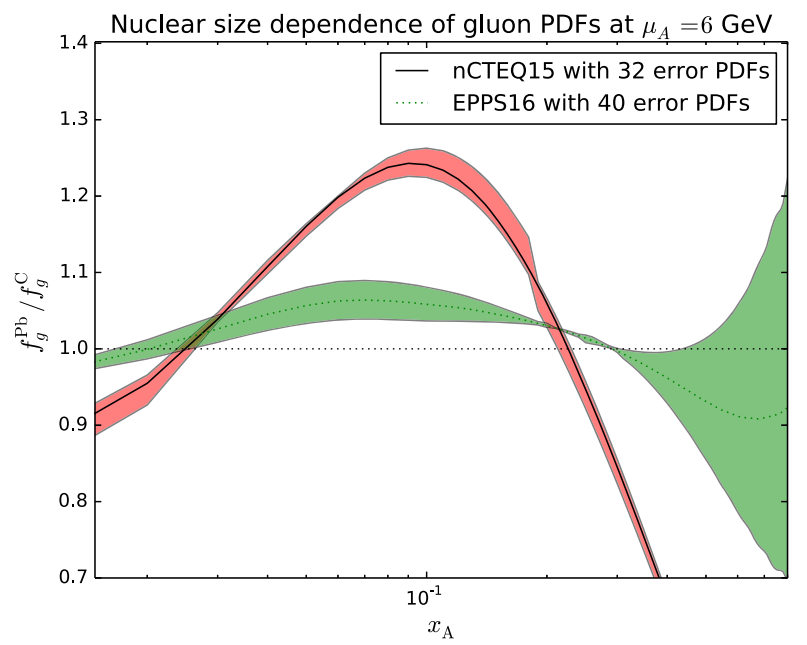

FIG. 5. Ratios of nuclear PDFs for Pb-208 over C-12 for up quarks (left) and gluons (right) at $\mu_{A}=6 \mathrm{GeV}$ using the nCTEQ15 (full black line) and EPPS16 (dotted green line) fits and their associated nuclear PDF uncertainties (red-shaded and green-shaded bands). 
constrain the up-quark PDFs relatively well, so that the shapes of the nCTEQ15 and EPPS16 ratios do not look too different, even though their error bands do not overlap in the region of intermediate $x_{A}$. The shapes for the gluon PDFs look, however, very different, and the error bands are largely underestimated due to theoretical bias in both fits. This situation therefore offers room for considerable improvement at an EIC that would be capable of colliding nuclei of different sizes.

\section{CONCLUSIONS AND OUTLOOK}

In conclusion, after an investigation of inclusive jet production in DIS [21], we have studied in this paper the potential of dijet photoproduction at the EIC to better constrain nuclear PDFs in the near future. We based our analysis on our theoretical framework of full NLO [4] and approximate NNLO QCD calculations [27], where we had found little impact of the aNNLO contributions on the central $K$-factors, but a sizable reduction of the scale uncertainty. The latter is also expected to cancel to a large extent in ratios of ion over free proton cross sections.

Because of the requirements of a minimum transverse momentum of 5 , not $4 \mathrm{GeV}$ and two jets, not only one, the kinematic reach was found to be somewhat smaller than in inclusive jet DIS. In particular, one cannot expect to reach $x$-values in the ion down to $2 \times 10^{-4}$ and scales up to $10^{3} \mathrm{GeV}^{2}$, but only $x$-values of $10^{-2}$ and $\bar{p}_{T}^{2}$ of $400 \mathrm{GeV}^{2}$. The jets would be well contained in a hadronic calorimeter with $\eta \in[-1 ; 3]$.

Despite the more limited kinematic reach, we found not only that one can probe the EMC and antishadowing regions but also that one can reach somewhat into the physically interesting and important shadowing region. Similar to our findings in DIS, EIC measurements have the potential to reduce the current theoretical uncertainty on nuclear PDFs by a factor of 10 to 5 , depending on how much information beyond DIS and DY has been included from existing hadron colliders.

The implementation of jet mass corrections [34] to our aNNLO formalism is left for future work. Although they will in particular introduce a dependence on the jet radius $R$, the impact of these additional corrections is expected to be even smaller than the one of the aNNLO contributions as a whole, in particular when $R=1$ as in this study, where terms $\ln R$ obviously disappear. Improvements similar to those at the EIC may also be expected from an $\mathrm{LHeC}$ [42]. Because of its potentially higher center-of-mass energy, the kinematic reach could even be larger there. Finally, even transverse-momentum dependent distribution functions of gluons in protons and nuclei might become accessible in measurements of dijet asymmetries in polarized or unpolarized $e p$ and $e A$ collisions at the EIC [43].

\section{ACKNOWLEDGMENTS}

We thank the organizers of the 8th International Conference on Physics Opportunities at an ElecTronIon-Collider (POETIC 8), which motivated this study, for the kind invitation and an anonymous referee for the suggestion to add Fig. 5. This work has been supported by the BMBF under Contract No. 05H15PMCCA. All figures have been produced using MATPLOTLIB [44].
[1] J. C. Collins, D. E. Soper, and G. F. Sterman, Adv. Ser. Dir. High Energy Phys. 5, 1 (1989).

[2] H. Abramowicz et al. (H1 and ZEUS Collaborations), Eur. Phys. J. C 75, 580 (2015).

[3] M. Klasen, G. Kramer, and B. Pötter, Eur. Phys. J. C 1, 261 (1998).

[4] M. Klasen and G. Kramer, Z. Phys. C 72, 107 (1996); 76, 67 (1997); M. Klasen, T. Kleinwort, and G. Kramer, Eur. Phys. J. direct C 1, 1 (2000).

[5] S. Dulat, T.-J. Hou, J. Gao, M. Guzzi, J. Huston, P. Nadolsky, J. Pumplin, C. Schmidt, D. Stump, and C.-P. Yuan, Phys. Rev. D 93, 033006 (2016); L. A. Harland-Lang, A. D. Martin, P. Motylinski, and R. S. Thorne, Eur. Phys. J. C 75, 204 (2015); R. D. Ball et al. (NNPDF Collaboration), Eur. Phys. J. C 77, 663 (2017).

[6] J. Butterworth et al., J. Phys. G 43, 023001 (2016).

[7] M. Hirai, S. Kumano, and T.-H. Nagai, Phys. Rev. C 76, 065207 (2007); M. Hirai, J. Phys. Soc. Jpn. Conf. Proc. 12, 010024 (2016); K. J. Eskola, H. Paukkunen, and
C. A. Salgado, J. High Energy Phys. 04 (2009) 065; D. de Florian, R. Sassot, P. Zurita, and M. Stratmann, Phys. Rev. D 85, 074028 (2012).

[8] N. Armesto, J. Phys. G 32, R367 (2006).

[9] L. Frankfurt, V. Guzey, and M. Strikman, Phys. Rev. D 71, 054001 (2005); A. J. Baltz et al., Phys. Rep. 458, 1 (2008); V. Guzey and M. Klasen, J. High Energy Phys. 04 (2016) 158.

[10] K. J. Golec-Biernat and M. Wüsthoff, Phys. Rev. D 59, 014017 (1998).

[11] F. Gelis, E. Iancu, J. Jalilian-Marian, and R. Venugopalan, Annu. Rev. Nucl. Part. Sci. 60, 463 (2010).

[12] K. Kovarik et al., Phys. Rev. D 93, 085037 (2016).

[13] M. Klasen and M. Brandt, Phys. Rev. D 88, 054002 (2013); M. Brandt, M. Klasen, and F. König, Nucl. Phys. A927, 78 (2014); A. Kusina, F. Lyonnet, D. B. Clark, E. Godat, T. Ježo, K. Kovařík, F. I. Olness, I. Schienbein, and J. Y. Yu, Eur. Phys. J. C 77, 488 (2017).

[14] N. Armesto, H. Paukkunen, J. M. Penn, C. A. Salgado, and P. Zurita, Eur. Phys. J. C 76, 218 (2016). 
[15] K. J. Eskola, P. Paakkinen, H. Paukkunen, and C. A. Salgado, Eur. Phys. J. C 77, 163 (2017).

[16] A. Kusina, J. P. Lansberg, I. Schienbein, and H. S. Shao, arXiv:1712.07024.

[17] B. Müller, in Proceedings of the 7th International Conference on Physics Opportunities at an ElecTron-IonCollider (POETIC 7), Philadelphia (Duke University and Brookhaven National Lab, Philadelphia, 2016).

[18] R. Yoshida, in Proceedings of the 7th International Conference on Physics Opportunities at an ElecTronIon-Collider (POETIC 7), Philadelphia (Jefferson Lab, Philadelphia, 2016).

[19] A. Accardi et al., Eur. Phys. J. A 52, 268 (2016).

[20] E. C. Aschenauer, S. Fazio, M. A. C. Lamont, H. Paukkunen, and P. Zurita, Phys. Rev. D 96, 114005 (2017).

[21] M. Klasen, K. Kovarik, and J. Potthoff, Phys. Rev. D 95, 094013 (2017).

[22] T. Biekötter, M. Klasen, and G. Kramer, Phys. Rev. D 92 , 074037 (2015).

[23] G. Abelof, R. Boughezal, X. Liu, and F. Petriello, Phys. Lett. B 763, 52 (2016).

[24] J. Currie, T. Gehrmann, and J. Niehues, Phys. Rev. Lett. 117, 042001 (2016).

[25] M. Klasen, Rev. Mod. Phys. 74, 1221 (2002).

[26] X. Chu, E. C. Aschenauer, J. H. Lee, and L. Zheng, Phys. Rev. D 96, 074035 (2017).

[27] M. Klasen, G. Kramer, and M. Michael, Phys. Rev. D 89, 074032 (2014).

[28] N. Kidonakis, Int. J. Mod. Phys. A 19, 1793 (2004).

[29] S. Frixione, M. L. Mangano, P. Nason, and G. Ridolfi, Phys. Lett. B 319, 339 (1993).
[30] M. Glück, E. Reya, and A. Vogt, Phys. Rev. D 46, 1973 (1992).

[31] M. Glück, E. Reya, and A. Vogt, Phys. Rev. D 45, 3986 (1992).

[32] F. Cornet, P. Jankowski, and M. Krawczyk, Phys. Rev. D 70, 093004 (2004).

[33] P. Aurenche, M. Fontannaz, and J. P. Guillet, Eur. Phys. J. C 44, 395 (2005).

[34] D. de Florian, P. Hinderer, A. Mukherjee, F. Ringer, and W. Vogelsang, Phys. Rev. Lett. 112, 082001 (2014).

[35] M. Cacciari, G. P. Salam, and G. Soyez, J. High Energy Phys. 04 (2008) 063.

[36] M. Klasen and G. Kramer, Phys. Lett. B 366, 385 (1996).

[37] D. F. Geesaman, K. Saito, and A. W. Thomas, Annu. Rev. Nucl. Part. Sci. 45, 337 (1995).

[38] S. J. Brodsky, I. Schmidt, and J. J. Yang, Phys. Rev. D 70, 116003 (2004); L. Frankfurt, V. Guzey, and M. Strikman, Phys. Rev. C 95, 055208 (2017).

[39] M. Arneodo, Phys. Rep. 240, 301 (1994).

[40] S. S. Adler et al. (PHENIX Collaboration), Phys. Rev. Lett. 98, 172302 (2007); B. I. Abelev et al. (STAR Collaboration), Phys. Rev. C 81, 064904 (2010).

[41] S. Chatrchyan et al. (CMS Collaboration), Eur. Phys. J. C 74, 2951 (2014).

[42] H. Paukkunen (LHeC study Group), Proc. Sci., DIS2017 (2018) 109.

[43] D. Boer, P. J. Mulders, C. Pisano, and J. Zhou, J. High Energy Phys. 08 (2016) 001.

[44] J. D. Hunter, Comput. Sci. Eng. 9, 90 (2007). 